\title{
An Inverse Economic Lot-sizing Approach to Eliciting Supplier Cost Parameters
}

\author{
Péter Egri ${ }^{1}$, Tamás Kis ${ }^{1}$, András Kovács $^{1, *}$, József Váncza $^{1,2}$ \\ ${ }^{1}$ Fraunhofer Project Center for Production Management and Informatics, \\ Institute for Computer Science and Control, Hungarian Academy of Sciences \\ ${ }^{2}$ Department of Manufacturing Science and Technology, \\ Budapest University of Technology and Economics, Hungary \\ \{egri,tamas.kis,andras.kovacs,vancza\}@sztaki.mta.hu
}

June 20, 2013

\begin{abstract}
Recent literature on supply chain coordination offers a wide range of game theoretic and optimization approaches that ensure efficient planning in the supply chain, but assume that the involved parties have complete information about each other. However, in reality, complete information is rarely available, and those models alone do not present any incentives for the parties to reveal their private information, e.g., the cost parameters that they use when solving their planning problems.

This paper proposes an inverse lot-sizing model for eliciting the cost parameters of a supplier from historic demand vs. optimal delivery lotsize pairs, gathered during repeated earlier encounters. It is assumed that the supplier solves a single-item, multi-period, uncapacitated lot-sizing problem with backlogs to optimality to calculate its lot-sizes, and the buyer is aware of this fact. The inverse lot-sizing problem is reformulated to an inverse shortest path problem, which is, in turn, solved as a linear program. This model is used to compute the ratios of the supplier's cost parameters, i.e., the setup, the holding, and the backlog cost parameters consistent with all the historic samples.

The elicited cost parameters can be used as input for various game theoretic or bilevel optimization models for supply chain coordination. Computational experiments on randomly generated problem instances indicate that the approach is very efficient in predicting future supplier actions from the historic records.
\end{abstract}

Keywords: Economic lot-sizing, inverse combinatorial optimization, eliciting cost parameters.

\footnotetext{
${ }^{*}$ Corresponding author
} 


\section{Introduction}

Planning inventories in a supply chain necessarily calls for the interaction of autonomous partners operating with distinct, potentially conflicting objectives, different decision mechanism and asymmetric information. Satisfying external demand requires the interaction of these partners in the supply chain. The literature offers a wide spectrum of coordination mechanisms $[2,28]$ based on game theoretic and optimization approaches, which make different assumptions on the information available to the different partners. Nevertheless, there is a considerable gap between the incomplete information models, which usually assume a single encounter of the buyer and the supplier with some well-defined asymmetric information situation, and the complete information models, which consider that the companies are mutually aware of their partners' decision situation. Namely, in case of repeated encounters, a significant amount of information is hidden in historic records of earlier interactions. These records can contain earlier orders, delivery lot-sizes, or delivery lead times. Furthermore, by the widespread application of tracking and tracing systems [17, 18], the buyer can observe even the production lot-sizes and the manufacturing parameters applied by the supplier. Exploiting this information enables a company to use well-informed, e.g., Stackelberg or bilevel optimization approaches for planning its production and logistics, providing a considerable competitive advantage compared to using models with restricted information.

In this paper, we tackle the issue of how the historic records of earlier encounters between a buyer and a supplier can be utilized in decision making. We take the stance of the buyer and aim at eliciting the cost parameters of the rational supplier's decision problem. It is assumed that the buyer possesses a historic record of demand vs. delivery lot-size pairs. It is noted that the same approach could be used for eliciting the supplier's cost parameters in its production lotsizing problem, given that the production lot-sizes are observed. We introduce an inverse combinatorial approach to eliciting the cost parameters of a supplier who determines its delivery periods and quantities by solving a single-item, multi-period, uncapacitated lot-sizing problem with backlogs (ULSB). Specifically, the proposed model computes the ratios of the supplier's setup, holding, and backlog cost parameters. It is noted that eliciting the absolute values of the cost parameters from the above input data is impossible, since the optimal delivery lot-sizes according to the ULSB model are invariant to the multiplication of the cost parameters by a common constant. To the best of our knowledge, this is the first inverse lot-sizing model investigated in the literature.

The elicited cost parameters can be useful in various scenarios involving a buyer-supplier relationship. A specific application is the utilization of the elicited cost parameters as inputs to one of the recent Stackelberg or bilevel approaches to lot-sizing in supply chains [20]. Such models require the knowledge of the supplier's cost parameters, but the coordination mechanisms themselves do not present any incentives for the supplier to reveal their true values. It is emphasized that in the above applications, eliciting the ratios of the cost parameters is sufficient, since, likewise the ULSB problem, the rational actions 
of the parties are insensible to the multiplication of the cost parameters by a common constant. Hence, our method can be a precious complement of those supply chain coordination approaches. On the other hand, a shortcoming of the approach is that it cannot compute the absolute values of the cost parameters, which can be an important limitation in other applications, e.g., in price negotiations.

In what follows, the related literature is surveyed first (Section 2). Then, the problem is defined formally and the inverse optimization solution method is introduced (Section 3). Next, the results of computational experiments are presented (Section 4), and finally, the paper is concluded with a discussion of the application opportunities and the directions for future research (Section 5).

\section{Literature review}

\subsection{Lot-sizing}

Fundamental results on dynamic lot-sizing models have been published in [30, 37]. These papers consider uncapacitated lot-sizing models where the deterministic, time varying demand is known in advance over a finite planning horizon. Over the past decades the basic models have been extended by production capacities and various side constraints, for an overview see, e.g., [3, 24, 25]. The modeling of various features in lot-sizing by mixed-integer programs (MIP) are investigated, e.g., in $[4,10]$.

The need for studying the interacting lot-sizing decisions of multiple autonomous parties in a supply chain is widely recognized. One of the possible approaches is integration, when the different parties jointly solve the interrelated planning problems, see, e.g., [21]. A drawback of integration is the mutual sharing of all the planning relevant information, which is sometimes unrealistic. A game theoretic approach alleviates this burden by using coordination mechanisms between the parties to drive the supply chain towards a system-wide optimal performance $[2,6]$. Four different computational approaches (decentralized planning, integration, coordination, and bilevel optimization) to the same lot-sizing problem in a two-player supply chain are compared in [20].

\subsection{Game theory}

Most papers in supply chain research assume complete information, i.e., that the game structure is common knowledge for the players [33]. In realistic situations however, there is an information gap between them-typically concerning either the cost structure or the demand forecast — which justifies the application of incomplete information (also called asymmetric information) models. Such approaches usually necessitate Bayesian setting, where the players have some common belief about the private information of the others. The inverse lotsizing model presented in this paper provides a similar sort of information to the underinformed player by characterizing the feasible cost parameters. The 
key difference between the two approaches is that we determine only the range of the feasible values, in contrast to the probability distribution computed by the Bayesian models. For further details on game theoretic models and their applications in inventory management problems, we refer to [33].

In two-player games with incomplete information, the process of learning the private information of the other player is called screening. Such models are widespread in the supply chain management literature, for a recent overview see [29]. For instance, Corbett [9] considers the case where either the setup cost or the backorder cost of the supplier is a private information. Xu et al. [34] present a model where the supplier's cost - which is inversely proportional to the required delivery time - is only known by the supplier, which is an obstacle for the buyer in optimizing its purchasing. Wang et al. [31] investigate situations where it is beneficial for the supplier to share its production cost information with the buyer truthfully. The two-player setting is more exhaustively investigated in [15], which also assumes that the price- and marketing-dependent demand rate is a private information of the buyer. We have studied an extended newsvendor type model in [14], where the buyer has private information about the uncertain demand forecast, while the supplier knows the various cost factors. The suggested coordination protocol and payment scheme provide both partners the right incentive for minimizing the total cost: the buyer is interested in sharing its unbiased information on the demand forecast and its uncertainty, while the supplier's rational decision concurs with the overall optimum.

A different approach to mitigating the effects of information asymmetry and ensuring a win-win situation for the players is called collaborative planning. In this case the goal is not eliciting the missing information, but the cooperative iterative improvement of the supply chain plan by non-hierarchical players. For uncapacitated dynamic lot-sizing in assembly networks Chu and Leon [8] present an iterative planning procedure. For the finite capacity case Dudek and Stadtler [13] developed a solution. A general overview of the collaborative planning problem can be found in [27].

\subsection{Inverse combinatorial optimization}

Inverse combinatorial optimization is a relatively new field of operations research. A comprehensive survey of this topic, including the studied problem models and algorithms has been given in [16]. Most of the previous work in the field focused on graph theoretical problems, such as the inverse shortest path problem [5] or the inverse center location problem [7]. A generic optimization model for a class of inverse problems has been introduced in [38], together with a Newton-type algorithm that runs in strongly polynomial time under mild conditions. In [1], it is shown that the inverse of a linear programming problem (LP) under the $L_{1}$ or the $L_{\infty}$ norm is also an LP, and polynomial-time algorithms are derived for the inverse problems of various classes of polynomially solvable combinatorial problems. On the other hand, extensions of these narrow-sense inverse optimization problems can easily become NP-hard, as it is shown in [36] for problems where only a partial solution is given in the input. 
To the best of our knowledge, this paper is the first to propose an inverse lotsizing model. However, there are a few earlier contributions that can be regarded as applications of inverse lot-sizing to very specific cases. For instance, various earlier papers present necessary or sufficient conditions for the optimality of the lot-for-lot policy for different lot-sizing models, see, e.g., [22, 32].

A closely related field of operations research is bilevel programming. It addresses decision and optimization problems whose outcome is determined by the interplay of two self-interested decision makers who decide sequentially, and whose pay-off functions mutually depend on the decision of the other party. The basic modeling and solution techniques in bilevel programming are presented in [12], while results in bilevel inventory control include [11, 26, 35].

The inverse lot-sizing problem model introduced in this paper is partly motivated by our bilevel lot-sizing model presented in [19]. The current inverse model and the follower's optimality condition in the bilevel model are based on a common idea, and an upfront application of the inverse model is to compute the supplier's cost parameters for the bilevel problem.

\section{An inverse optimization approach to cost elic- itation}

\subsection{Problem definition}

A problem of cost elicitation in a dyadic supply chain, consisting of a single buyer and a single supplier is investigated. The buyer aims at eliciting the cost parameters of its supplier from historic records of earlier encounters between the two parties. The historic records consist of a set of $M$ samples. Each sample contains a vector of demand values, $d_{i}^{m}$, over a horizon of $T$ time periods, and a vector of corresponding delivery lot-sizes, $x_{i}^{m}, m=1 \ldots M, i=1 \ldots T$. The following assumptions are made:

- The supplier determines the delivery lot-sizes $x_{i}^{m}$ by solving a single-item uncapacitated lot-sizing problem with backlogs (ULSB) to optimality over the demand $d_{i}^{m}$ submitted by the buyer.

- The supplier uses the same combination of cost parameters, i.e., the setup cost $f_{0}$, the holding cost $h_{0}$, and the backlog cost $g_{0}$, to determine its lot-sizes in all samples.

- In case the ULSB problem has multiple optimal solutions for a given demand $d_{i}^{m}$, the supplier may choose an arbitrary optimal solution.

- The buyer is aware of the ULSB decision framework of the supplier. However, the cost parameters of the supplier, i.e., $f_{0}, h_{0}$, and $g_{0}$, are unknown to the buyer.

- The delivery lot-sizes applied by the supplier are observed by the buyer.

Possible relaxations of these assumptions are discussed in Section 5. 


\subsubsection{The direct ULSB problem}

Before presenting the inverse lot-sizing problem investigated in the paper, let us recapitulate the direct ULSB problem. The input of ULSB consists of the demand values, $d_{i}$, over a horizon of $T$ time periods, and three cost parameters, namely the setup cost, $f$, a per period and per unit holding cost, $h$, and a per period and per unit backlog cost, $g$. It is assumed that all cost parameters are non-negative real numbers. The objective is to determine the delivery lot-sizes, $x_{i}, i=1 \ldots T$ in such a way that all demand is satisfied over the time horizon and the total cost is minimized. Using the auxiliary, implied variables $s_{i}$ for stock quantities, $r_{i}$ for backlogs, and $y_{i}$ for setup events, the (direct) ULSB problem can be stated as follows:

$\operatorname{ULSB}(d, f, h, g)$ :

$$
\text { Minimize } \sum_{i=1}^{T}\left(f y_{i}+h s_{i}+g r_{i}\right)
$$

subject to

$$
\begin{array}{ll}
x_{i}+\left(r_{i}-r_{i-1}\right)=d_{i}+\left(s_{i}-s_{i-1}\right) & i=1, \ldots, T \\
x_{i} \leq D y_{i} & i=1, \ldots, T \\
s_{0}=s_{T}=r_{0}=r_{T}=0 & \\
x_{i}, r_{i}, s_{i} \geq 0 & i=1, \ldots, T \\
y_{i} \in\{0,1\} & i=1, \ldots, T
\end{array}
$$

The objective (1) is minimizing the total cost, composed of the setup, the holding, and the backlog costs. Constraints (2) ensure the inventory balance, while inequality (3) states that the item can be produced in a given period only if a setup is performed in that period. Constant $D$ is the total demand, i.e., $D=\sum_{i=1}^{T} d_{i}$. Constraints (4) set the initial/final stock/backlog to zero, which implies that the total demand will be met throughout the planning horizon.

In the sequel, the combination of cost parameters $(f, h, g)$ used by the supplier to solve its direct ULSB problem will be denoted by $\left(f_{0}, h_{0}, g_{0}\right)$.

\subsubsection{The inverse ULSB problem}

The focus of the current paper is the inverse $U L S B$ problem, where the objective is eliciting the values of $f, h$, and $g$ from a set of $M$ corresponding demand vs. optimal delivery lot-size pairs, $\mathcal{X}=\left\{\left(d^{m}, x^{m}\right), m=1, \ldots, M\right\}$. As stated above, it is assumed that each lot-size vector $x^{m}=\left(x_{1}^{m}, \ldots, x_{T}^{m}\right)$ is an optimal solution of the corresponding direct ULSB problem with demand vector $d^{m}=$ $\left(d_{1}^{m}, \ldots, d_{T}^{m}\right)$. First, it should be observed that, in general, the samples do not determine the values of $(f, h, g)$ unambiguously. Therefore, the solution of the inverse ULSB problem is a range of feasible combinations of the cost parameters $(f, h, g)$, denoted as $S(\mathcal{X})$ :

$$
S(\mathcal{X})=\left\{(f, h, g) \mid x^{m} \in O p t\left(U L S B\left(d^{m}, f, h, g\right)\right) \forall m=1, \ldots, M\right\},
$$


where $\operatorname{Opt}(U L S B()$.$) stands for the set of optimal solutions of the direct ULSB$ problem with the given input parameters. However, the set $\operatorname{Opt}(U L S B()$.$) is$ invariant to the multiplication of the cost parameters, i.e., for a given demand vector $d_{i}^{m}$ and cost parameters $(f, h, g)$, it holds that $\operatorname{Opt}\left(U L S B\left(d^{m}, f, g, h\right)\right)=$ $\operatorname{Opt}\left(U L S B\left(d^{m}, c f, c h, c g\right)\right)$, where $c$ is an arbitrary positive constant. This implies that the approach is unsuitable for finding the absolute values of $(f, h, g)$; only their ratios can be determined. Accordingly, in the sequel we investigate an equivalent problem in which $f$ is fixed to a positive constant. To avoid scaling problems, the setup cost value used by the supplier, $f_{0}$, will be applied:

$$
S(\mathcal{X})=\left\{(h, g) \mid x^{m} \in \operatorname{Opt}\left(U L S B\left(d^{m}, f=f_{0}, h, g\right)\right) \forall m=1, \ldots, M\right\} .
$$

Note that the range $S(\mathcal{X})$ is a region of the two dimensional $(h, g)$ plane. However, for reasons of computational efficiency, instead of characterizing $S(\mathcal{X})$ exactly, we resort to determining its bounding box, $B(\mathcal{X})$, which is defined by the four values $h_{\min }, h_{\max }, g_{\min }$, and $g_{\max }$ :

$$
B(\mathcal{X})=\left\{(h, g) \mid h_{\min } \leq h \leq h_{\max } \wedge g_{\min } \leq g \leq g_{\max }\right\}
$$

where

$$
\begin{aligned}
h_{\min } & =\min \left\{h \mid \exists g \forall m: x^{m} \in \operatorname{Opt}\left(U L S B\left(m, f=f_{0}, h, g\right)\right)\right\}, \\
h_{\max } & =\max \left\{h \mid \exists g \forall m: x^{m} \in \operatorname{Opt}\left(U L S B\left(m, f=f_{0}, h, g\right)\right)\right\}, \\
g_{\min } & =\min \left\{g \mid \exists h \forall m: x^{m} \in \operatorname{Opt}\left(U L S B\left(m, f=f_{0}, h, g\right)\right)\right\}, \\
g_{\max } & =\max \left\{g \mid \exists h \forall m: x^{m} \in \operatorname{Opt}\left(U L S B\left(m, f=f_{0}, h, g\right)\right)\right\}
\end{aligned}
$$

The range of feasible values, $S(\mathcal{X})$, and it bounding box, $B(\mathcal{X})$, is displayed in the $(h, g)$ plane for two different problem instances in Figure 1. The left hand side of the figure presents a case where $S(\mathcal{X})$ is bounded by finite $h_{\max }$ and $g_{\max }$ values. The original values of the cost parameters, used for generating the samples, were $h_{0}=82$ and $g_{0}=133$, while the proposed approach elicited the bounding box defined by $h \in[71.43,96.15], g \in[112.90,136.99]$ from the 5 samples included in the input. Detailed data for this problem instance is presented in Appendix A. In the right had side of the figure, $S(\mathcal{X})$ is displayed for a set of samples that does not contain any backlogging action. Hence, the samples do not imply any finite bound $g_{\max }$; they may even belong to a problem where backlogging is not allowed (equivalent to $g=\infty$ ). It is noted that while no finite $h_{\max }$ or $g_{\max }$ may exist, $S(\mathcal{X})$ is always bounded from below by finite values $h_{\min } \geq 0$ and $g_{\min } \geq 0$.

\subsection{A linear programming formulation}

The reformulation of the inverse optimization problem to a linear program (LP) is based on the idea of regeneration intervals [37] and a standard shortest path representation of the ULSB problem [19, 23]. Namely, any instance of the ULSB problem admits an optimal solution in which the time horizon can be subdivided into a series of regeneration intervals $[i, k]$, such that all demand in the interval 

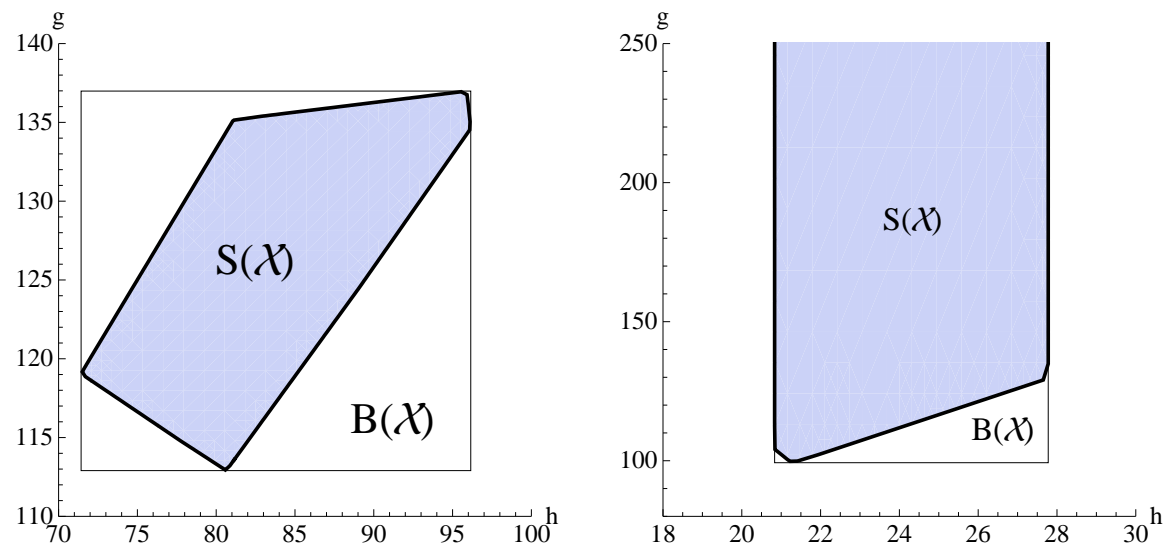

Figure 1: The feasible range $S(\mathcal{X})$ and its bounding box $B(\mathcal{X})$ for two problem instances, each containing 5 samples. The range on the left is bounded by finite $h_{\max }$ and $g_{\max }$ values (the supplier's original cost parameters were $h_{0}=82$ and $\left.g_{0}=133\right)$. The range on the right is unbounded $\left(g_{\max }=\infty\right)$, since the corresponding samples did not contain any backlogging action $\left(h_{0}=25\right.$ and $\left.g_{0}=187\right)$.

$[i, k]$ is met by production in a single period $j$ with $i \leq j \leq k$, i.e., $x_{j}^{m}=$ $\sum_{\ell=i}^{k} d_{\ell}^{m}$. Therefore, $d^{m}$ and $x^{m}$ uniquely determine the series of regeneration intervals in sample $m$ (apart from time periods with $d_{\ell}=0$, which can be assigned to the previous or the subsequent regeneration interval arbitrarily).

Then, the graph representation of our inverse problem consists of $M$ disjoint components for the $M$ samples. Let us denote the component corresponding to sample $m$ by $G^{m}$. There are $T+1$ nodes in $G^{m}$, standing for time periods $0,1, \ldots, T$. Between any pair of nodes $i, k$ of $G^{m}$ with $i<k$, there are $k-i$ parallel directed edges pointing from node $i$ to node $k$, which correspond to a possible regeneration interval $[i+1, k]$ and the $k-i$ possible positions of the single production period in the interval. The length of edge $(m, i, j, k)$ is denoted by $c_{i j k}^{m}$, and it is the sum of the setup cost plus the total holding and backlogging cost incurred if production occurs in period $j$ to meet all the demand in the regeneration interval $[i+1, k]$, where $i<j \leq k$.

Hence,

$$
c_{i j k}^{m}=f+\sum_{u=i+1}^{j-1}(j-u) g d_{u}^{m}+\sum_{u=j+1}^{k}(u-j) h d_{u}^{m} .
$$

In order to maintain the distance of a node from the first node of the corresponding component, potential values $\pi_{j}^{m}$ are assigned to each node. The potential values are consistent with the edge lengths if and only if $c_{i j k}^{m} \geq \pi_{k}^{m}-\pi_{i}^{m}$ holds for each edge, and $c_{i j k}^{m}=\pi_{k}^{m}-\pi_{i}^{m}$ for the edges of the shortest path. This graph representation is illustrated in Figure 2.

Then, the optimal solution of the ULSB corresponding to sample $m$ is en- 


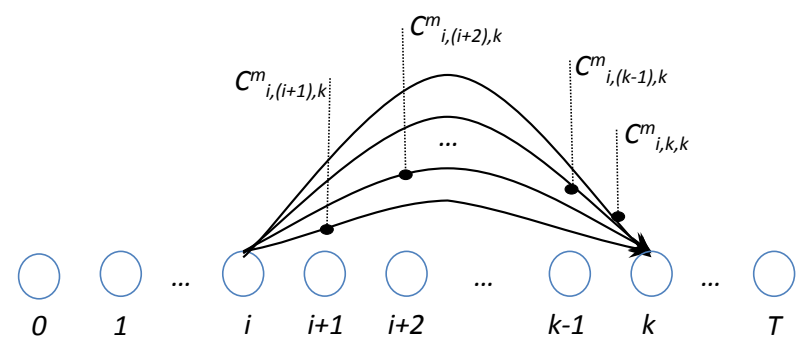

Figure 2: Graph representation of the ULSB problem. The figure displays the $T+1$ nodes of $G^{m}$, the graph component corresponding to sample $m$. The $k-i$ parallel edges between nodes $i$ and $k$, with the displayed edge weights, stand for the possible locations of the single production period in the candidate regeneration interval $[i+1, k]$.

coded in the shortest path between the first and last nodes of $G^{m}$. Notice that the optimal ULSB solutions, and therefore the set of all edges along the corresponding shortest paths are now given in the input of our inverse problem. Let us denote this set of edges by $S P$. Now, we are ready to define our LP formulation of the problem:

$$
\text { Minimize or maximize } \quad h \text { or } g
$$

subject to

$$
\begin{array}{ll}
c_{i j k}^{m}-\pi_{k}^{m}+\pi_{i}^{m}=0 & \forall(m, i, j, k) \in S P \\
c_{i j k}^{m}-\pi_{k}^{m}+\pi_{i}^{m} \geq 0 & \forall(m, i, j, k) \notin S P \\
c_{i j k}^{m}=f+\sum_{u=i}^{j-1}(j-u) g d_{u}^{m}+\sum_{u=j+1}^{k}(u-j) h d_{u}^{m} & \forall m, i, j, k \\
\pi_{0}^{m}=0 & \forall m \\
c_{i j k}^{m}, \pi_{k}^{m}, h, g \geq 0 & \forall m, i, j, k
\end{array}
$$

In this LP formulation, the variables are the cost parameters $h$ and $g$, as well as the edge lengths $c_{i j k}^{m}$ and potential values $\pi_{i}^{m}$. Constraints (11) and (12) ensure the consistency of the edge lengths and node potentials with the shortest paths encoded in $S P$. Constraint (13) defines the edge lengths, while lines (14) and (15) set the ranges of the variables.

Lemma $1 S(\mathcal{X})$ is the projection of the feasible region of (11)-(15) onto the variables $(h, g)$.

Proof: For all $m=1, \ldots, M$, the values $x^{m}$ constitute an optimal solution of the supplier with parameters $h$ and $g$ if and only if the path encoded by $S P$, corresponding to $x^{m}$, is a shortest path in $G^{m}$. On the other hand, the wellknown linear programming formulation of the shortest path problem (11)-(15) 
ensures the latter property [23]. Hence, $(h, g, c, \pi)$ is a feasible solution of (11)(15) if and only if $S P$ is a shortest path for $h$ and $g$.

From the above lemma it follows that, in order to solve the inverse ULSB problem (9), the LP model (10)-(15) must be solved four times with four different criteria:

- with the criterion of minimizing $h$ for determining $h_{\min }$;

- maximizing $h$ for computing $h_{\max }$;

- minimizing $g$ for determining $g_{\min }$;

- and maximizing $g$ for calculating $g_{\max }$.

\subsection{Key characteristics}

Below we present key properties of the proposed approach to solving the inverse ULSB problem.

Property 1 The feasible range, $S(\mathcal{X})$, is non-empty, since the supplier's true cost parameter combination is a feasible solution, i.e., $\left(h_{0}, g_{0}\right) \in S(\mathcal{X})$.

Property 2 The feasible range, $S(\mathcal{X})$, is a (bounded or unbounded) convex polygon in the $(h, g)$ plane.

Proof: Lemma 1 states that $S(\mathcal{X})$ is the projection of the feasible region of the LP (11)-(15) onto variables $(h, g)$. The projection of a convex polytope on some of the coordinates is a convex polygon, from which the above property follows directly. The polygon is bounded from below by $h_{\min } \geq 0$ and $g_{\min } \geq 0$. On the other hand, it may or may not be bounded from above by a finite $h_{\max }$ and $g_{\max }$. More specifically, $h_{\max }$ is finite if and only if at least one sample $m$ contains a period $i$ where a positive amount stock $s_{i}^{m}$ is kept. Similarly, $g_{\max }$ is finite if and only if there exists $m$ and $i$ such that $r_{i}^{m}>0$. Otherwise, the polygon is unbounded (see Figure 1 for illustrations).

Property 3 If $h_{\max }=\infty$ and $g_{\max }=\infty$, then for any $h^{\prime} \geq h_{0}$ and $g^{\prime} \geq g_{0}$, $\left(h^{\prime}, g^{\prime}\right) \in S(\mathcal{X})$.

Proof: Let $x^{m}$ denote the lot-size vector in sample $m, x^{m^{\prime}}$ an arbitrary ULSB solution for the same demand vector, $h^{\prime} \geq h_{0}$, and $g^{\prime} \geq g_{0}$. Furthermore, let $C\left(x^{m^{\prime}}, h^{\prime}, g^{\prime}\right)$ denote the cost of $x^{m^{\prime}}$ with cost parameters $h^{\prime}$ and $g^{\prime}$. Now, assume that $h_{\max }=\infty$ and $g_{\max }=\infty$. Then, $s_{i}^{m}=r_{i}^{m}=0, \forall i, m$. We will show that

$$
C\left(x^{m}, h^{\prime}, g^{\prime}\right)=C\left(x^{m}, h_{0}, g_{0}\right) \leq C\left(x^{m^{\prime}}, h_{0}, g_{0}\right) \leq C\left(x^{m^{\prime}}, h^{\prime}, g^{\prime}\right)
$$


The first equality holds because $C\left(x^{m}, h^{\prime}, g^{\prime}\right)$ is composed of the setup costs $f_{0}$ for periods $i$ with $x_{i}^{m}>0$ only, which is independent of $h^{\prime}$ and $g^{\prime}$. The inequality in the middle encodes the optimality of $x^{m}$ with $\left(h_{0}, g_{0}\right)$. The last inequality holds because increasing the cost parameters $h^{\prime}$ or $g^{\prime}$ cannot decrease the monotonous objective function for a fixed $x^{m^{\prime}}$. From the above series of inequalities it follows that $C\left(x^{m}, h^{\prime}, g^{\prime}\right) \leq C\left(x^{m \prime}, h^{\prime}, g^{\prime}\right)$, which proves the optimality of $x^{m}$ for $h^{\prime}$ and $g^{\prime}$, i.e., $\left(h^{\prime}, g^{\prime}\right) \in S(\mathcal{X})$.

The above property characterizes the shape of unbounded feasible regions. It expresses that if $S(\mathcal{X})$ is unbounded from the upper left diagonal direction, then it is unbounded from above and from the left as well. It is noted that an analogous proof can be given based on the LP (11)-(15). The proof consists of a construction scheme for the variables of the LP that allows increasing $h$ arbitrarily, while keeping $g$ fixed (or vice versa) and maintaining the feasibility of the LP.

Property 4 The feasible range, $S(\mathcal{X})$, does not necessary contain any of the corners of its bounding box $B(\mathcal{X})$. This property has been proven by computing the polygon defined by $S(\mathcal{X})$ on a sample instance, displayed in Figure 1.

Property 5 The following combination of cost parameters is a feasible solution of the inverse lot-sizing problem, i.e., $\left(h^{\prime}, g^{\prime}\right) \in S(\mathcal{X})$ :

$$
\begin{aligned}
& h^{\prime}= \begin{cases}\infty & \text { if } h_{\max }=\infty \\
\frac{h_{\min }+h_{\max }}{2} & \text { otherwise }\end{cases} \\
& g^{\prime}= \begin{cases}\infty & \text { if } g_{\max }=\infty \\
\frac{g_{\min }+g_{\max }}{2} & \text { otherwise }\end{cases}
\end{aligned}
$$

With finite $h_{\max }$ and $g_{\max }$, the property can be justified by a simple geometrical proof, since any convex shape contains the mid-point of its bounding box. On the other hand, $h_{\max }=\infty$ (respectively, $g_{\max }=\infty$ ) if and only if holding stocks (resp., backlogging) never occurs in the samples, which is again consistent with the above $h^{\prime}$ and $g^{\prime}$. As a special case, $h_{\max }=\infty$ and $g_{\max }=\infty$ at the same time means that the supplier delivered on a lot-for-lot basis in the samples. The significance of this property is given by applications where a specific, feasible combination of cost parameters is required, instead of the range or the bounding box.

Property 6 The bounding box $B(\mathcal{X})$ can be computed in polynomial time in the number of samples and in the length of the time horizon. This follows from the fact that the problem can be formulated as a series of four LP problems, with sizes bounded by a polynomial function of those two parameters. 
Property 7 If, for a given combination of parameters, the direct $U L S B\left(d^{m}, f, h, g\right)$ problem has multiple optimal lot-size vectors, then $S(\mathcal{X})$ and $B(\mathcal{X})$ depend on which optimal lot-size vector $x^{m}$ is included in $\mathcal{X}$. However, for any choice of optimal lot-size vector, it is guaranteed that $\left(h_{0}, g_{0}\right) \in S(\mathcal{X})$. This property follows directly from Property 1.

\section{Experiments}

\subsection{Accuracy of the parameter licitation}

The first set of computational experiments addressed the accuracy of the elicited cost parameters, including the dependence of the accuracy on various factors, such as the number of samples, $M$, the length of the time horizon, $T$, or the characteristics of the instances. Two types of problem instances have been generated: in the independent samples instances, the samples were generated using independent random demand, $d_{i}^{m} \leftarrow U[1,10]$, where $U[a, b]$ denotes the integer uniform random distribution over the interval $[a, b]$. In the rolling horizon instances, the demand in the first sample, $x_{i}^{1}$ was generated in a similar fashion, whereas subsequent demand vectors were generated by shifting the previous demand earlier by one period, and perturbing the demand value by at most $10 \%$, i.e., $d_{i}^{m+1}=d_{i+1}^{m}(U[90,110] / 100)$. The supplier's original setup cost parameter was fixed to $f_{0}=1000$, while the holding and backlogging costs were randomized, $h_{0} \leftarrow U[10,100]$ and $g_{0} \leftarrow U[20,200]$. Obviously, these cost parameters were only used for generating the samples, and were not included in the input of the inverse problem. The lot-sizes in the samples were generated by solving the standard MIP formulation of the ULSB problem presented above. Two different values were considered for the length of the time horizon, $T=10$ and $T=25$. 100 instances were generated for each combination of the instance type and the length of the time horizon, with 50 historic samples in each instance.

The experiments were performed on an implementation of the LP formulation in FICO Xpress 7.2 using the Mosel programming language. All instances could be solved to optimality in less than one second, i.e., the exact values of $h_{\min }, h_{\max }, g_{\min }$, and $g_{\max }$ could be computed. For this reason, the solution times are not displayed below. We recall that the proposed approach elicits the ratios of the cost parameters, i.e., $h / f$ and $g / f$. Nevertheless, to facilitate the evaluation of the results in terms of $h$ and $g$, below we use the $f_{0}=1000$ for scaling them.

Figure 3 shows the results achieved for an independent samples instance, with $T=10$, originally generated with $h=36$ and $g=89$. The diagram on the left displays the computed bounds of the holding cost parameter, while the diagram on the right refers to the backlog cost parameter. The horizontal axis shows the number of samples, $M$, applied for the parameter elicitation. The maximum and minimum cost parameter curves indicate that the accuracy of the elicitation improved gradually, in multiple steps, until the end of the elicitation process. Finally, after 50 samples, the bounds 35.71-36.52 were computed for 

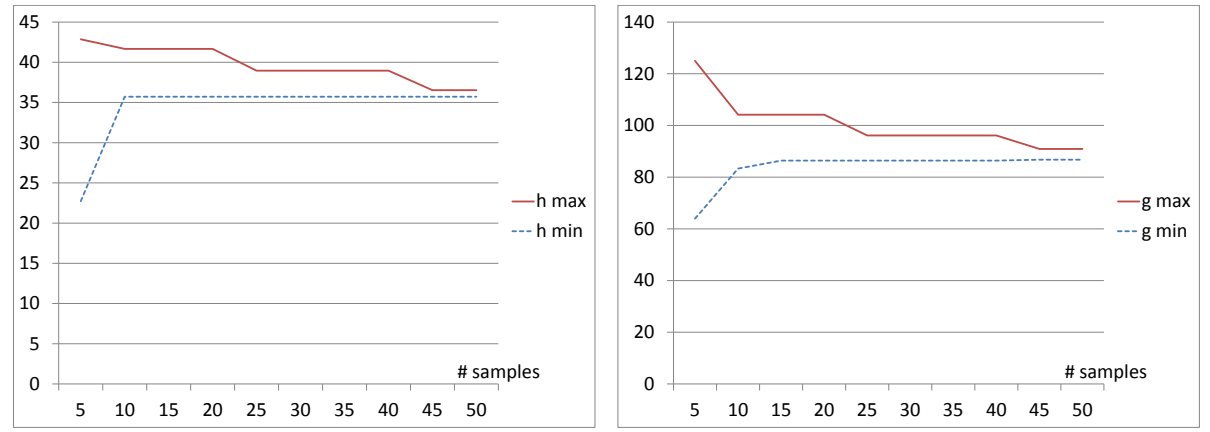

Figure 3: The elicited bounds of the holding cost $h$ (left) and the backlog cost $g$ (right) as a function of the number of samples, $M$, used for the elicitation on a selected problem instance.

$h$ (corresponding to a gap of 2.27\%) and the bounds 86.76-90.91 for $g(4.78 \%)$. Expectably, the gaps would reduce further if more samples were considered.

The summary of the experimental results on the 200 sample instances with $T=10$ is presented in Table 1 . The sub-tables on the left display the results for the elicitation of the holding cost parameter, $h$, for the independent samples and the rolling horizon instances, respectively. The rows of the sub-tables correspond to different ranges of $h$ (the value used when generating the samples). The ranges were determined in such a way that each row contains data for 25 instances. The columns of the tables stand for the different values of $M \in\{10,25,50\}$. Each cell contains the average gap measured on the 25 corresponding instances, where the gap was computed as $\frac{h_{\max }-h_{\min }}{h_{\min }} \cdot 100 \%$ for each individual instance.

The sub-tables on the right display the results for the backlog cost parameter, $g$. The table design is similar to the one above, except that the rows correspond to different ranges of $g$. The gap associated to the elicited $g$ was calculated as $\frac{g_{\max }-g_{\min }}{g_{\min }} \cdot 100 \%$. The numbers in parentheses indicate the number of instances where this gap was infinitely large. This occurred when the samples used for the elicitation did not contain any backlogging action, and therefore, no finite $g_{\text {max }}$ could be computed.

The results show that the average gap decreased continuously with increasing $M$, and in the end it reached $4.70-8.64 \%$ for parameter $h$ and $5.90-15.73 \%$ for parameter $g$. We note that $h$ and $g$ have symmetric roles in the model, but the different random distributions used for generating the instances caused a difference in the accuracy for $h$ and $g$. The best accuracy for $h$ was achieved when $h$ was relatively low, but not extremely low. This occurs because for high values of $h$, stocking actions appear too rarely in the ULSB solution to determine $h_{\max }$ precisely. On the contrary, for extremely low $h$, a stocking action is taken in almost all time units, which implies that few data is available for bounding $h_{\min }$. The case is similar for $g$. Furthermore, the elicitation is somewhat more accurate on independent samples than on the rolling horizon 
instances, since the samples in the latter case are interrelated, and therefore contain fewer information.

The results with $T=25$ are displayed in Table 2, in a table structure similar to the one above. The different values of the $M \in\{4,10,20\}$ were determined in such a way that the total volume of input data matches the data volume of the corresponding instances with $T=10$ (e.g., 4 samples with $T=25$ versus 10 samples with $T=10$ ). These results suggest the same general conclusions as those with $T=10$; independent samples lead to significantly better accuracy than rolling horizon samples, and increasing $M$ improves the accuracy drastically, reaching $4.17-13.31 \%$ for $h$ and $5.64-41.47 \%$ for $g$ with $M=20$. Nevertheless, a detailed comparison of the figures with $T=10$ and those with $T=25$ shows a substantial difference of the achieved accuracy on the same amount of data, depending on the length of the time horizon. Eliciting $h$ from the independent samples instances with $T=25$ and $M=M_{0}$ resulted in slightly better accuracy than with $T=10$ and $M=2.5 M_{0}(10.93 \%$ versus $11.98 \%$ on average). This supports the natural conclusion that, for a given volume of historic data, better accuracy can be achieved with higher $T$ and lower $M$ than with lower $T$ and higher $M$.

However, the case of rolling horizon instances is substantially different. Here, $T=25$ and $M=M_{0}$ leads to significantly worse accuracy than $T=10$ and $M=2.5 M_{0}$ (22.44\% versus $16.27 \%$ on average $)$. This can be attributed to the fact that the historic data in a rolling horizon instance is highly redundant. It encodes the behavior of the supply channel on an interval of $T+M-1$ time units, which determines the value of information in the overall data volume of $O(T M)$. In our case, this leads to more information in the instances with $T=10$ than with $T=25$, especially with higher values of $M$. Similar observations can be made for the elicitation of parameter $g$.

It is noted that the two types of problem instances, the independent samples and the rolling horizon instances model the two extremities of the interrelation among the samples, i.e., independent and tightly related samples. For a degree of correlation between these two extremities, e.g., for rolling horizon instances with a perturbation above $10 \%$, the accuracy can be expected to be in between the accuracies achieved with the two sets of instances investigated here.

\subsection{Prediction of future supplier actions}

The second set of experiments investigated the possibility of using the inverse optimization approach to predict the future actions of the supplier. For this purpose, the proposed method was used to elicit cost parameters from $M$ samples (with $M=10,25,50$ ), resulting in cost parameters $h^{\prime}$ and $g^{\prime}$, as defined by Property 5 . The elicited cost parameters were used, in turn, to predict the delivery lot-sizes for the demand vector in a subsequent, $(M+1)$ th test sample. Then, the predicted lot-sizes (i.e., the optimal ULSB solutions with the elicited parameters, $h^{\prime}$ and $g^{\prime}$ ) were compared to the actual lot-sizes (i.e., optimal ULSB solutions with the supplier's original parameters, $h_{0}$ and $\left.g_{0}\right)$. Whenever multiple optimal ULSB solutions existed, one was chosen randomly. The prediction was 


\begin{tabular}{|r|r|r|r|}
\hline \multicolumn{4}{|c|}{$T=10$, independent samples, $h$} \\
\hline & \multicolumn{3}{|c|}{ No. of samples } \\
$h$ & 10 & 25 & 50 \\
\hline $12-32$ & 28.67 & 11.97 & 5.85 \\
$33-54$ & 17.84 & 8.47 & 4.70 \\
$60-80$ & 18.82 & 10.26 & 6.21 \\
$81-100$ & 14.42 & 9.38 & 7.16 \\
\hline \hline \multicolumn{4}{|c|}{. 10 , rolling horizon, $h$} \\
\hline \multicolumn{4}{|c|}{ No. samples } \\
$h$ & 10 & 25 & 50 \\
\hline $10-33$ & 30.33 & 12.93 & 6.69 \\
$35-56$ & 21.71 & 8.58 & 6.26 \\
$57-83$ & 31.24 & 12.56 & 7.84 \\
$85-99$ & 33.00 & 15.47 & 8.64 \\
\hline
\end{tabular}

\begin{tabular}{|c|c|c|c|}
\hline \multicolumn{4}{|c|}{$T=10$, independent samples, $g$} \\
\hline & \multicolumn{3}{|c|}{ No. of samples } \\
\hline$g$ & 10 & 25 & 50 \\
\hline $21-62$ & 25.42 & 10.93 & 6.04 \\
\hline $65-102$ & 24.66 & 11.60 & 5.90 \\
\hline $107-148$ & 44.03 & 15.70 & 7.71 \\
\hline $153-196$ & 83.10 & 46.72 & 14.38 \\
\hline \multicolumn{4}{|c|}{$T=10$, rolling horizon, $g$} \\
\hline & \multicolumn{3}{|c|}{ No. of samples } \\
\hline$g$ & 10 & 25 & 50 \\
\hline $23-65$ & 26.19 & 12.76 & 7.19 \\
\hline $70-116$ & 38.82 & 16.14 & 8.82 \\
\hline $117-155$ & 41.01 & 17.96 & 10.01 \\
\hline $159-200$ & 122.09 & 42.52 & 15.73 \\
\hline
\end{tabular}

Table 1: Accuracy of the elicitation with $T=10$, in percent. The tables display the average accuracy for $h$ (left) and $g$ (right), for different cost parameters and numbers of samples. The numbers in parentheses indicate the number of instances where the gap between the lower and upper bounds was infinitely large.

accepted to be correct if the predicted and the actual lot-sizes matched perfectly. Three different types of problem instances were used for the experiment:

- independent samples for elicitation and for testing;

- a series of rolling horizon samples for elicitation, plus an independent sample for testing; and

- rolling horizon samples for elicitation and testing, where the test sample is the last member of the series of samples generated by the rolling horizon generation scheme presented above.

It is noted that since a rolling horizon test sample is reasonable only with rolling horizon samples for elicitation, no further combinations are possible. All other parameters of the instances were the same as for the previous set of experiments.

The experimental results are displayed in Table 3, which shows the percentage of correctly predicted lot-sizes for the three sets of instances and for different values of $M$, with $T=10$. The results show that the approach could give correct predictions in up to $98 \%$ of the cases (using 50 samples and rolling horizon samples for elicitation and testing). However, the performance of the approach depends both on $M$ and on the type of the instances. The predictions were correct for $93-98 \%$ of the instances when 50 samples were available for elicitation, but only for $85-93 \%$ when just 10 samples could be used. Furthermore, the prediction is better when the elicitation is performed on independent samples (93-97\%, depending on $M$ ), rather than on rolling horizon samples (85-93\%). It should be emphasized that the difference diminishes gradually as $M$ increases (difference of $8 \%$ for 10 samples, and $4 \%$ for 50 samples). On the contrary, better results can be achieved when the test sample is correlated to the samples 


\begin{tabular}{|r|r|r|r|}
\hline \multicolumn{4}{|c|}{$T=25$, independent samples, $h$} \\
\hline & \multicolumn{3}{|c|}{ No. of samples } \\
$h$ & 4 & 10 & 20 \\
\hline $10-38$ & 18.51 & 7.17 & 4.17 \\
$38-54$ & 16.87 & 7.69 & 5.33 \\
$55-80$ & 17.76 & 9.45 & 7.28 \\
$80-100$ & 19.27 & 10.86 & 6.82 \\
\hline \multicolumn{4}{|c|}{$T=25$, rolling horizon, $h$} \\
\hline \multicolumn{4}{|c|}{ No. of samples } \\
$h$ & 4 & 10 & 20 \\
\hline $11-29$ & 33.97 & 15.85 & 9.95 \\
$29-48$ & 32.97 & 17.22 & 9.87 \\
$48-74$ & 38.1 & 23.79 & 12.1 \\
$74-100$ & 39.55 & 22.62 & 13.31 \\
\hline
\end{tabular}

\begin{tabular}{|r|rr|r|r|}
\hline \multicolumn{5}{|c|}{$T=25$, independent samples, $g$} \\
\hline \multicolumn{5}{|c|}{ No. of samples } \\
\hline $20-71$ & 22.99 & & 9.31 & 20 \\
$74-119$ & 27.26 & & 12.85 & 5.64 \\
$120-155$ & 25.28 & $(3)$ & 14.21 & 7.58 \\
$158-200$ & 46.94 & $(3)$ & $27.93 \quad(2)$ & 20.64 \\
\hline \hline \multicolumn{5}{|c|}{$T=25$, rolling horizon, $g$} \\
\hline \multicolumn{5}{|c|}{ No. of samples } \\
\hline $23-66$ & 34.85 & & 10 & 20 \\
$68-103$ & 46.65 & $(1)$ & 30.88 & 11.04 \\
$106-147$ & 72.67 & $(2)$ & 42.37 & 24.21 \\
$149-199$ & 84.59 & $(6)$ & 63.58 & 41.47 \\
\hline
\end{tabular}

Table 2: Accuracy of the elicitation with $T=25$, in percent. The tables display the average accuracy for $h$ (left) and $g$ (right), for different cost parameters and numbers of samples. The numbers in parentheses indicate the number of instances where the gap between the lower and upper bounds was infinitely large.

\begin{tabular}{|l|l|ccc|}
\hline \multicolumn{2}{|c|}{ No. of samples } & 10 & 25 & 50 \\
\hline Samples for elicitation & Test sample & & & \\
\hline Independent & Independent & $93 \%$ & $94 \%$ & $97 \%$ \\
Rolling horizon & Independent & $85 \%$ & $90 \%$ & $93 \%$ \\
Rolling horizon & Rolling horizon & $91 \%$ & $96 \%$ & $98 \%$ \\
\hline
\end{tabular}

Table 3: Percentage of correctly predicted supplier actions for different numbers of samples.

used for elicitation due to the rolling horizon generation scheme (correct prediction for $91-98 \%$ of the instances, depending on $M$ ) than when the test sample is independent (85-93\%). However, the difference in this case does not decrease rapidly with the increase of $M$ (difference of $6 \%$ and $5 \%$ for 10 and 50 samples).

\section{Conclusions and future work}

This paper proposed a novel technique for eliciting a supplier's cost parameters from earlier records of supply request vs. delivery lot-size pairs by using an inverse optimization approach. An inverse ULSB lot-sizing model, and its reformulation to a linear program was introduced. The approach was evaluated in computational experiments, where the cost parameters could be elicited with an accuracy of $5-16 \%$, whereas future supplier actions could be predicted with a success rate up to $98 \%$ from 50 samples, with a planning horizon of 10 time units. We consider that the achieved precision is sufficient in most supply chain applications.

The above model can be useful in various scenarios involving a buyer-supplier 
relationship. Beyond the obvious and general benefit of knowing the partner's cost parameters, the approach enables the use of complete information models to supply chain coordination even when a part of the required information is not explicitly available, but it is encoded in historic records about earlier encounters of the parties. A specific application derives from the numerous recent cooperation mechanism for lot-sizing in supply chains based on Stackelberg games. Such models assume that the buyer knows the cost parameters of the supplier, but the coordination mechanisms themselves do not present any incentives for the supplier to reveal the true values of its cost parameters. Hence, the proposed approach can be regarded as a precious complement of recent game theoretic and bilevel approaches to coordinating supply chains. On the other hand, the approach elicits only the ratios of the different cost parameters, not their absolute values, which can be an important limitation in some applications, e.g., for price negotiations.

The most important direction for future work is extending the approach to handle noisy samples, i.e., allowing the supplier to slightly deviate from its optimal ULSB solutions. Another interesting direction is the extension of the model towards richer lot-sizing models. Some extensions, e.g., costs varying over time, can be easily added to the mathematical model, but the resulting high number of free decision variables, and in turn, different feasible solutions, make it complicated to achieve useful results. Other extensions, such as finite capacities, require changing the core mathematical model as well. Our longterm objective is the composition of a portfolio of inverse lot-sizing models, which is applicable to eliciting also the lot-sizing model applied by the supplier. The key idea is performing the elicitation using each model in the portfolio, on samples regarded as noisy. The model that fits with the least noise is accepted as the model applied by the supplier.

\section{Acknowledgements}

The authors are grateful to the anonymous referees for their constructive comments that helped improve the content of the paper. This work has been supported by the OTKA grant K76810 and the NKTH grant OMFB-01638/2009. P. Egri acknowledges the support of the János Bolyai scholarship no. BO/00659/11/6.

T. Kis is grateful for the support of the János Bolyai scholarship no. BO/00412/12/3.

\section{References}

[1] R. K. Ahuja and J. B. Orlin. Inverse optimization. Operations Research, 49(5):771-783, 2001.

[2] M. Albrecht. Supply Chain Coordination Mechanisms. Springer, 2010.

[3] S. Axsäter. Inventory Control. Springer, 2nd edition, 2006. 
[4] G. Belvaux and L. A. Wolsey. Modelling practical lot-sizing problems as mixed-integer programs. Management Science, 47(7):993-1007, 2001.

[5] D. Burton and Ph. L. Toint. On an instance of the inverse shortest paths problem. Mathematical Programming, 53:45-61, 1992.

[6] G. P. Cachon. Supply chain coordination with contracts. In A. G. de Kok and S. C. Graves, editors, Supply Chain Management: Design, Coordination and Cooperation, volume 11 of Handbooks in Operations Research and Management Science, pages 229-339. Elsevier, 2003.

[7] M. C. Cai, X. G. Yang, and J. Z. Zhang. The complexity analysis of the inverse center location problem. Journal of Global Optimization, 15:213218, 1999.

[8] C.-L. Chu and V.J. Leon. Scalable methodology for supply chain inventory coordination with private information. European Journal of Operational Research, 195(1):262-279, 2009.

[9] C.J. Corbett. Stochastic inventory systems in a supply chain with asymmetric information: Cycle stocks, safety stocks, and consignment stock. Operations Research, 49(4):487-500, 2001.

[10] C. Cordier, H. Marchand, R. Laundy, and L. A. Wolsey. bc-opt: a branchand-cut code for mixed integer programs. Mathematical Programming, Ser. A, 86:335-353, 1999.

[11] A. G. de Kok and G. Muratore. Coordinating supply chains: a bilevel programming approach. In International Workshop on Supply Chain Models for Shared Resource Management, 2010.

[12] S. Dempe. Foundations of Bilevel Programming. Kluwer, 2002.

[13] G. Dudek and H. Stadtler. Negotiations-based collaborative planning between supply chain partners. European Journal of Operational Research, 163:668-687, 2005.

[14] P. Egri and J. Váncza. Channel coordination with the newsvendor model using asymmetric information. International Journal of Production Economics, 135(1):491-499, 2012.

[15] M. Esmaeili and P. Zeephongsekul. Seller-buyer models of supply chain management with an asymmetric information structure. International Journal of Production Economics, 123(1):146-154, 2010.

[16] C. Heuberger. Inverse combinatorial optimization: A survey on problems, methods, and results. Journal of Combinatorial Optimization, 8:329-361, 2004 . 
[17] J. Holmström, K. Främling, and T. Ala-Risku. The uses of tracking in operations management: Synthesis of a research program. International Journal of Production Economics, 126(2):267-275, 2010.

[18] E. Ilie-Zudor, Zs. Kemény, F. van Blommestein, L. Monostori, and A. van der Meulen. A survey of applications and requirements of unique identification systems and RFID techniques. Computers in Industry, $62(3): 227-252,2011$.

[19] T. Kis and A. Kovács. Exact solution approaches for bilevel lot-sizing. European Journal of Operational Research, 226(2):237-245, 2013.

[20] A. Kovács, P. Egri, T. Kis, and J. Váncza. Inventory control in supply chains: Alternative approaches to a two-stage lot-sizing problem. International Journal of Production Economics, 143(2):385-394, 2013.

[21] X. Li and Q. Wang. Coordination mechanisms of supply chain systems. European Journal of Operational Research, 179(1):1-16, 2007.

[22] C. L. Munson and M. J. Rosenblatt. Coordinating a three-level supply chain with quantity discounts. IIE Transactions, 33:371-384, 2001.

[23] C. H. Papadimitriou and K. Steiglitz. Combinatorial Optimization: Algorithms and Complexity. Dover, 1998.

[24] Y. Pochet. Mathematical programming models and formulations for deterministic planning problems. In M. Jünger and D. Naddef, editors, Computational Combinatorial Optimization, Lecture Notes in Computer Science, volume 2241, pages 57-111. Springer, 2001.

[25] Y. Pochet and L. A. Wolsey. Production planning by mixed integer programming. Spinger, 2006.

[26] J-H. Ryu, V. Dua, and E. N. Pistikopoulos. A bilevel programming framework for enterprise-wide process networks under uncertainty. Computers and Chemical Engineering, 28:1121-1129, 2004.

[27] H. Stadtler. A framework for collaborative planning and state-of-the-art. OR Spectrum, 31:5-30.

[28] J. Váncza, L. Monostori, D. Lutters, S.R.K. Kumara, M. Tseng, P. Valckenaers, and H. Van Brussel. Cooperative and responsive manufacturing enterprises. CIRP Annals - Manufacturing Technology, 60(2):797-820, 2011.

[29] G. Voigt. Supply Chain Coordination in Case of Asymmetric Information. Springer, 2011.

[30] H. M. Wagner and T. M. Whitin. Dynamic version of the economic lot size model. Management Science, 5(1):89-96, 1958. 
[31] J.-C. Wang, H.-S. Lau, and A.H.H. Lau. When should a manufacturer share truthful manufacturing cost information with a dominant retailer? European Journal of Operational Research, 197(1):266-286, 2009.

[32] Z. K. Weng. Modeling quantity discounts under general price-sensitive demand functions: Optimal policies and relationships. European Journal of Operational Research, 86:300-3014, 1995.

[33] H. Wu and M. Parlar. Games with incomplete information: A simplified exposition with inventory management applications. International Journal of Production Economics, 133(2):562-577, 2011.

[34] H. Xu, N. Shi, S. Ma, and K.K. Lai. Contracting with an urgent supplier under cost information asymmetry. European Journal of Operational Research, 206(2):374-383, 2010.

[35] W. Yang, L. Li, and S. Ma. Coordinating supply chain response-time: a bi-level programming approach. International Journal of Advanced Manufacturing Technology, 31:1034-1043, 2007.

[36] X. Yang. Complexity of partial inverse assignment problem and partial inverse cut problem. RAIRO Operations Research, 35(1):117-126, 2001.

[37] W. I. Zangwill. A backlogging model and a multi-echelon model of a dynamic economic lot size production system - a network approach. Management Science, 15(9):506-527, 1969.

[38] J. Zhang and Z. Liu. A general model of some inverse combinatorial optimization problems and its solution method under $l_{\infty}$ norm. Journal of Combinatorial Optimization, 6:207-227, 2002.

\section{Appendix A: A sample problem}

Below we present the detailed data of the sample problem related to the left hand side of Figure 1. The length of the time horizon was $T=10$ periods, and only $M=5$ samples were considered. The cost parameters used by the supplier to solved the direct ULSB problem were as follows:

$$
\begin{array}{lll}
f_{0}=1000 & h_{0}=82 & g_{0}=133
\end{array}
$$

The demand values $d_{i}^{m}$, generated randomly by the method presented in Section 4 , and the delivery lot-sizes $x_{i}^{m}$ that were computed by solving the direct ULSB problem (1)-(6) are presented in Table 4. The data contained in the table constitutes the input for the inverse ULSB problem.

Given this input data, the proposed approach to the inverse ULSB problem consists of solving four copies of the LP formulation (10)-(15), with the four different optimization criteria. Solving the LP with the criterion of minimizing $h$ results in $h=71.43$ (which defines $h_{\min }$ ) and $g=119.05$. The values for the 


\begin{tabular}{|l|rrrrrrrrrr|}
\hline$i$ & 1 & 2 & 3 & 4 & 5 & 6 & 7 & 8 & 9 & 10 \\
\hline$d_{i}^{1}$ & 7 & 8 & 0 & 9 & 10 & 6 & 9 & 6 & 5 & 3 \\
$x_{i}^{1}$ & 15 & 0 & 0 & 9 & 16 & 0 & 15 & 0 & 8 & 0 \\
\hline$d_{i}^{2}$ & 5 & 4 & 10 & 6 & 0 & 10 & 8 & 3 & 2 & 3 \\
$x_{i}^{2}$ & 9 & 0 & 16 & 0 & 0 & 18 & 0 & 0 & 8 & 0 \\
\hline$d_{i}^{3}$ & 4 & 2 & 1 & 9 & 6 & 9 & 5 & 8 & 1 & 10 \\
$x_{i}^{3}$ & 6 & 0 & 0 & 16 & 0 & 14 & 0 & 9 & 0 & 10 \\
\hline$d_{i}^{4}$ & 6 & 7 & 1 & 7 & 10 & 1 & 10 & 5 & 6 & 4 \\
$x_{i}^{4}$ & 13 & 0 & 0 & 18 & 0 & 0 & 16 & 0 & 10 & 0 \\
\hline$d_{i}^{5}$ & 5 & 4 & 6 & 3 & 8 & 7 & 0 & 9 & 3 & 8 \\
$x_{i}^{5}$ & 0 & 15 & 0 & 0 & 18 & 0 & 0 & 12 & 0 & 8 \\
\hline
\end{tabular}

Table 4: The set of historic samples $\mathcal{X}$ used as the input for the inverse ULSB problem: demand vectors $d_{i}^{m}$ and lot-sizes $x_{i}^{m}$. The shading of the rows reflects the regeneration intervals.

auxiliary variables $\pi_{i}^{m}$ and $c_{i j k}^{m}$ are presented in Table 5 . Note that displaying the complete matrix $c_{i j k}^{m}$ would require excessive space, therefore only the values $c_{i j k}^{m}$ that denote edge lengths along the shortest path, i.e., $(m, i, j, k) \in S P$, are presented. The values of both $\pi_{i}^{m}$ and $c_{i j k}^{m}$ are different when the LP is solved with a different optimization criterion.

\begin{tabular}{|l|rrrrrrrrrr|}
\hline$i$ & 1 & 2 & 3 & 4 & 5 & 6 & 7 & 8 & 9 \\
\hline$\pi_{i}^{1}$ & 1000.00 & 1571.43 & 1571.43 & 2571.43 & 3285.71 & 4000.00 & 4857.14 & 5428.57 & 5642.86 & 6642.86 \\
$\pi_{i}^{2}$ & 1000.00 & 1285.71 & 2285.71 & 2714.29 & 2714.29 & 3714.29 & 4285.71 & 4642.86 & 4857.14 & 5857.14 \\
$\pi_{i}^{3}$ & 1000.00 & 1142.86 & 1261.90 & 1976.19 & 2690.48 & 3452.38 & 4047.62 & 5023.81 & 5119.05 & 6119.05 \\
$\pi_{i}^{4}$ & 1000.00 & 1500.00 & 1619.05 & 2404.76 & 3333.33 & 3476.19 & 4214.29 & 4809.52 & 5095.24 & 6095.24 \\
$\pi_{i}^{5}$ & 1000.00 & 1285.71 & 2023.81 & 2380.95 & 2880.95 & 3880.95 & 3880.95 & 4738.10 & 5095.24 & 6095.24 \\
\hline$c^{1}$ & $c_{0,1,3}^{1}=1571.43$ & $c_{3,4,4}^{1}=1000.00$ & $c_{4,5,6}^{1}=1428.57$ & $c_{6,7,8}^{1}=1428.57$ & $c_{8,9,10}^{1}=1214.29$ \\
$c^{2}$ & $c_{0,1,2}^{2}=1285.71$ & $c_{2,3,5}^{2}=1428.57$ & $c_{5,6,7}^{2}=1571.43$ & $c_{7,9,10}^{2}=1571.43$ & & \\
$c^{3}$ & $c_{0,1,2}^{3}=1142.86$ & $c_{2,4,5}^{3}=1547.62$ & $c_{5,6,7}^{3}=1357.14$ & $c_{7,8}^{3}=1071.43$ & $c_{9,10,10}^{3}=1000.00$ \\
$c^{4}$ & $c_{0,1,2}^{4}=1500.00$ & $c_{2,4,5}^{4}=1833.33$ & $c_{5,7,8}^{4}=1476.19$ & $c_{8,10}^{4}=1285.71$ & & \\
$c^{5}$ & $c_{0,2,3}^{5,}=2023.81$ & $c_{3,5,7}^{5}=1857.14$ & $c_{7,8,9}^{5}=1214.29$ & $c_{9,10,10}^{5}=1000.00$ & & \\
\hline
\end{tabular}

Table 5: Values of variables $\pi_{i}^{m}$ and $c_{i j k}^{m}$ in the solution of the LP for minimizing $h$. Only the values $c_{i j k}^{m}$ that denote edge lengths along the shortest path, i.e., $(m, i, j, k) \in S P$ are presented. It can be observed that $c_{i j k}^{m}-\pi_{k}^{m}+\pi_{i}^{m}=0$ holds for all the displayed values, as requested by constraint (11).

Solving the LP with all the four possible optimization criteria results in the following bounding box, $B(\mathcal{X})$, for the feasible combinations of cost parameters (scaled with $f_{0}=1000$ ):

$$
\begin{aligned}
h_{\min } & =71.43 & h_{\max } & =96.15 \\
g_{\min } & =112.90 & g_{\max } & =136.99
\end{aligned}
$$

Note that the feasible range of cost parameter combinations, $S(\mathcal{X})$, for this problem instance has been displayed earlier in Figure 1. 
According to Lemma $1, S(\mathcal{X})$ is the projection of the feasible region of the LP (11)-(15) onto the variables $(h, g)$. Since the projection of a linear program onto a subset of variables is computationally expensive (the FourierMotzkin elimination is of exponential running time in the length of the LP), the displayed range has been computed using an approximation method. Namely, a tangent of the convex polygon $S(\mathcal{X})$ has been determined from each possible direction, with a resolution of 1 degree. This has been performed by solving the LP (11)-(15) with the objective function of minimizing $\cos \varphi \cdot h+\sin \varphi \cdot g$ for $\varphi=1^{\circ}, 2^{\circ}, \ldots, 360^{\circ}$.

The following observations can be made. The elicited bounds of the cost parameters are correct, since the original cost parameters $h_{0}$ and $g_{0}$ fall into these intervals. The corresponding gaps are $34.6 \%(h)$ and $21.3 \%(g)$, which are rather wide. On the other hand, when solving the same problem (i.e., using the same $f_{0}, h_{0}$, and $\left.g_{0}\right)$ with $M=50$ independent random samples, the accuracy of the elicitation improved drastically, leading to gaps $4.5 \%(h)$ and $3.4 \%(g)$. 